PHYSICAL REVIEW D 83, 035020 (2011)

\title{
Atomic precision tests and light scalar couplings
}

\author{
Philippe Brax* \\ Institut de Physique Théorique, CEA, IPhT, CNRS, URA2306, F-91191 Gif-sur-Yvette cédex, France
}

Clare Burrage ${ }^{\dagger}$

Départment de Physique Théorique, Université de Genève, 24 Quai E. Ansermet, CH-1211, Genève, Switzerland

Theory Group, Deutsches Elektronen-Synchrotron DESY, D-22603, Hamburg, Germany

(Received 3 November 2010; published 25 February 2011)

\begin{abstract}
We calculate the shift in the atomic energy levels induced by the presence of a scalar field which couples to matter and photons. We find that a combination of atomic measurements can be used to probe both these couplings independently. A new and stringent bound on the matter coupling springs from the precise measurement of the $1 s$ to $2 s$ energy level difference in the hydrogen atom, while the coupling to photons is essentially constrained by the Lamb shift. For a range of masses these constraints are not as stringent as those from fifth force experiments or optical astrophysical and laboratory measurements. However, they have the advantage that they are universal, applying to all scalars, even those that hide their effects dynamically from fifth force searches, such as the chameleon and Galileon models. Combining these constraints with current particle physics bounds we find that the contribution of a scalar field to the recently claimed discrepancy in the proton radius measured using electronic and muonic atoms is negligible.
\end{abstract}

DOI: 10.1103/PhysRevD.83.035020

PACS numbers: 31.10.+z, 14.80.-j, 31.30.jr, 36.10.Ee

\section{INTRODUCTION}

Scalar fields coupled to matter occur in a wide variety of fundamental contexts: The inflationary scalar field should couple to matter in order to reheat the Universe, and such a coupling seems likely to exist for the dark energy scalar field too. Indeed most attempts at modifying gravity, or unifying it with particle physics, predict new scalars coupling to the standard model particles in the form of nonrenormalizable interactions that are suppressed by the energy scale characteristic of the energies probed by the model. For example, many coupled scalars are present in the four-dimensional effective theories arising from compactifying the extra dimensions of string theory [1-3].

It is well known that light canonical scalar fields which couple to matter are tightly constrained by experimental searches for fifth forces and violations of the equivalence principle [4], although nonlinear effects such as in the chameleon [5,6] or Galileon [7] cases allow the fields to easily avoid these constraints through dynamical mechanisms. In this article we do not restrict ourselves to light fields, specifying only that the field should not be so heavy that its mass would lie above the cutoff of the low energy effective field theory we wish to study. These will be predominately atomic experiments for which we study an effective field theory valid up to a cutoff high enough to include effects from the standard model of particle physics. Therefore we consider the effects of a theory in which particles with masses $\gtrsim \mathrm{TeV}$ have been integrated out.

\footnotetext{
*philippe.brax@cea.fr

† clare.burrage@unige.ch
}

We will find that the approximations required to obtain constraints mean that they will only apply to scalars with masses $\lesssim 10 \mathrm{keV}$. The existence of very light scalars with fixed mass $\lesssim 10^{-3} \mathrm{eV}$ and fixed coupling to matter are excluded by searches for fifth forces [8]. However, as these constraints can be avoided by nonlinear mechanisms such as those of the chameleon and Galileon theories we consider it important to obtain constraints which apply universally to all scalar fields.

The phenomenology of such fields is not restricted to their effects in gravitational experiments. The minimal scenario we consider is that the scalar field couples to matter conformally, i.e. through a scalar field dependent conformal rescaling of the metric. Classically a conformal coupling means that the scalar field couples to fermions but not to massless bosons such as the photon. It was shown in $[9,10]$, however, that, given a conformal coupling, quantum effects lead to a coupling between photons and scalars in the low energy effective theory. As already mentioned, in this article we focus our attention on low energy atomic experiments which we consider to be described by low effective theories with a cutoff $\sim \mathrm{TeV}$. We include a coupling of the scalar field to photons as a generic property of the low energy theory.

It is also possible to study the effects of coupled scalar fields both in particle colliders and in low energy, high precision experiments. If the scalar couples to photons, it has a phenomenology similar to the Peccei-Quinn axion; in particular, oscillations between photons and scalars can occur in the presence of magnetic fields [11]. Much effort has gone into exploring the consequences of such couplings both in the laboratory [12-18] and in astrophysics 
[19-26]. For light particles with fixed masses $m_{\phi} \lesssim$ $10^{-3} \mathrm{eV}$ and couplings these observations are extremely constraining forcing the energy scale of the coupling of the scalar to photons to be $\gtrsim 10^{10} \mathrm{GeV}$. However, these constraints are also easily avoided in nonlinear models [27] and so in this article we focus on constraints which are universal to all models. A scalar field coupled to the fields of the standard model has also direct effects on the properties of these particles-making the masses of elementary particles, and other energy scales of the theory, become dependent on the scalar field. Consequently the Hamiltonian describing the low energy behavior of any fermion becomes scalar field dependent. Previously [28] we have shown that this leads to electrons transitioning between energy levels in atoms in the presence of a background scalar condensate. This gives rise to new possibilities of searching for scalar fields via scalar field stimulated photon emission. In this paper we show that the presence of a scalar will also perturb the energy levels of atoms leading to new constraints on coupled scalar fields from precision atomic measurements.

The scalar field induced shift in energy levels can be constrained by measurements of the energy gap between the $1 s$ and $2 s$ orbitals of the hydrogen atom. A second consequence of the shifts in energy levels is a scalar field dependent change in the Lamb shift: the small difference in energy between the $2 s$ and $2 p$ energy levels of hydrogen caused by the interaction between the electron and the background [29]. We will show that the scalar field dependence of the Lamb shift, induced by a coupling between fermions and the scalar field, will lead to different values of the proton charge radius when measured with muons and with electrons. Comparison of properties of electronic and muonic atoms will be shown to be a sensitive probe of the existence of new scalar fields. The recently proposed existence of a possible $5 \sigma$ discrepancy between the proton charge radius [30] measured recently from the Lamb shift in muonic hydrogen (an atom formed by a proton and a negative muon), compared to that inferred previously from hydrogen atom spectroscopy, will lead to a new constraint on scalar couplings, i.e. an upper bound on the geometrical mean of the couplings to matter and photons.

Scalar fields are not the only new physics that can modify atomic spectra. The presence of additional, hidden sector, $U(1)$ gauge groups also gives rise to a change in the Lamb shift [31,32], as well as modifications of Coulomb's law which can be tested through atomic measurements.

In the following section, we recall standard properties of coupled scalars. In Sec. III, we calculate the effect of a scalar field on the atomic levels, with particular focus on the energy level difference between the $1 s$ and $2 s$ states of the hydrogen atom and the Lamb shift. The $1 s$ to $2 s$ energy difference leads to a new and stringent upper bound on the coupling of scalars to matter and the Lamb shift leads to a looser upper bound on the geometrical mean of the photon and matter couplings. We then combine these atomic constraints with those obtained from high energy particle physics experiments. We find that the upper bound on the matter coupling obtained from the $1 s$ to $2 s$ gap is stronger than the particle physics bounds. On the other hand, the constraint on the coupling to photons deduced from the width of the $Z$ boson and electroweak precision tests is more restrictive than atomic physics bounds. We then apply these results to the proton charge radius discrepancy and find that it is incompatible with the $Z$ width bound on the coupling of scalars to gauge fields. Hence scalar fields cannot provide an explanation to the proton radius anomaly. We conclude in Sec. IV.

\section{COUPLED SCALAR FIELDS}

We consider a scalar-tensor theory defined by the Lagrangian

$$
\begin{aligned}
S= & \int d^{4} x \sqrt{-g}\left(\frac{R}{2 \kappa_{4}^{2}}-\frac{1}{2}(\partial \phi)^{2}-V(\phi)\right) \\
& +\mathcal{L}_{m}\left(\Psi_{i}, A^{2}(\phi) g_{\mu \nu}\right),
\end{aligned}
$$

where the matter fields $\Psi_{i}$ feel a metric $\tilde{g}_{\mu \nu}=A^{2}(\phi) g_{\mu \nu}$. It is possible to allow the scalar to couple differently to different particle species [10]; however, we restrict ourselves here to a universal coupling which captures all the relevant phenomenology. Equation (1) is known as the Einstein action for a coupled scalar field; a conformal rescaling allows a classically equivalent description of the theory in which particle properties are independent of the scalar field, but the gravitational sector of the theory becomes scalar dependent, known as the Jordan frame description. For computational convenience we work in the Einstein frame in what follows. We consider that the Einstein frame theory (1) is an effective field theory valid up to an energy cutoff and that couplings given by nonrenormalizable operators are suppressed by powers of the cutoff scale.

In a nonrelativistic background the Klein-Gordon equation for the scalar field arising from (1) is modified and becomes

$$
D^{2} \phi=\frac{\partial V}{\partial \phi}+A(\phi) \rho
$$

where $\rho$ is the classical energy density. The dynamics of the field $\phi$ are determined by the effective potential

$$
V_{\mathrm{eff}}(\phi)=V(\phi)+\rho A(\phi) .
$$

We assume that this effective potential has a minimum at $\phi=\phi_{0}$ so that the field is stabilized.

In addition to the terms in Eq. (1) quantum effects will generate $[9,10]$ a term which describes the coupling of the scalar field to photons: 


$$
\mathcal{L}_{\gamma}=\frac{\phi}{2 M_{\gamma}} F^{\mu \nu} F_{\mu \nu}=\frac{\phi}{M_{\gamma}}\left(\mathbf{E}^{2}-\mathbf{B}^{2}\right),
$$

where $F_{\mu \nu}$ is the electromagnetic field tensor and $\mathbf{E}$ and $\mathbf{B}$ are the electric and magnetic fields, respectively. This contributes to the scalar field effective potential in a similar manner to the background matter energy density giving

$$
V_{\text {eff }}(\phi)=V(\phi)+\rho A(\phi)+\frac{\phi}{M_{\gamma}}\left(\mathbf{B}^{2}-\mathbf{E}^{2}\right) .
$$

From this point onwards we assume that $\phi_{0}$ minimizes the effective potential including the background contributions from the electric and magnetic fields.

In a homogeneous background the scalar field can be expanded about its minimum value $\phi_{0}$ :

$$
A(\phi)=A\left(\phi_{0}\right)\left(1+\frac{A^{\prime}\left(\phi_{0}\right)}{A\left(\phi_{0}\right)} \delta \phi+\cdots\right),
$$

where $\delta \phi$ is the fluctuation induced by the presence of a matter source. We assume that higher order terms in this expression are small and can be consistently neglected. We can write the coefficient of the second term as an inverse energy scale:

$$
\frac{A^{\prime}\left(\phi_{0}\right)}{A\left(\phi_{0}\right)}=\frac{1}{M_{m}} .
$$

We view (1) as a low energy effective theory and therefore expect $M_{m}$ and $M_{\gamma}$ to be of the order of the cutoff scale of the theory in the relevant energy range as it will be sensitive to fields that have been integrated out at higher energy. We will be interested in an effective low energy theory describing a muon, or an electron in the background of a hydrogen nucleus; therefore, we expect $M_{m}, M_{\gamma} \geqslant \mathrm{GeV}$, larger than the muon and proton masses. Particle physics effects at accelerators and therefore the effective theory involving $W$ bosons would require the coupling to $\phi$ to be determined by a cutoff scale larger than the mass of the $W$ boson. Constraints from particle physics impose that $M_{m}$ and $M_{\gamma}$ should be at least in the $\mathrm{TeV}$ and $M_{Z}$ ranges, respectively [33].

It has been recently argued [34] that the coupling of scalars to matter in scalar-tensor theories is preserved under renormalization and that the only effect of quantum corrections is to induce a change due to the wave function renormalization of the scalar field. When integrating out momenta to obtain the effective scalar theory valid at a lower energy scale, radiative corrections in the scalar sector imply that the wave function renormalization $Z_{\phi}$ should be affected by logarithmic terms and therefore

$$
\phi_{\text {low }}=Z_{\phi} \phi_{\text {high }}
$$

where $\phi_{\text {low }}$ is the normalized field after integration over momenta between the low energy and the high energy cutoffs. This leads to a direct relation between the coupling scale to matter at low and high energies:

$$
M_{m}^{\text {low }}=\frac{M_{m}^{\text {high }}}{Z_{\phi}} .
$$

Logarithmic corrections do not entail a large scale dependence of the coupling scale $M_{m}$.

Within the effective field theory approach used in this paper, the various coupling scales are just an effective parametrization which needs to be deduced from experiment. In the absence of an underlying theory going beyond the standard model coupled to scalars, we have simply given a useful parametrization of the scalar-matter coupling and its phenomenological consequences in atomic physics.

The coupling of the scalar field to fermions implies that the fermion masses $m_{f}$ become scalar field dependent:

$$
m_{f}(\phi)=A(\phi) m_{f 0}
$$

where $m_{0}$ is the bare mass as it appears in the Lagrangian. Again expanding around the background value of the scalar field we find

$$
m_{f}(\phi)=m_{f}\left(1+\frac{\delta \phi}{M_{m}}\right) .
$$

We assume that the higher order terms in this expression can be consistently neglected, and we have normalized $A\left(\phi_{0}\right)=1$. Notice that, viewed as a low energy operator, the fermionic mass term $m_{f}(\phi) \bar{\psi} \psi$ is a nonrenormalizable effective interaction term. When truncating this interaction to first order in $\phi$ it reduces to an effective Yukawa interaction with a coupling $m_{f} / M_{m}$ which must be small as the lepton of mass $m_{f}$ has not been integrated out.

\section{ATOMIC ENERGY SHIFTS}

In and around atoms the scalar field perturbation is sourced by the presence of the nuclear electric field

$$
\mathbf{E}=\frac{Z e \mathbf{r}}{4 \pi r^{3}},
$$

in cgs units, implying a perturbation to the effective potential (4):

$$
\delta V=-\phi \frac{Z^{2} \alpha}{4 \pi M_{\gamma} r^{4}},
$$

and by the pointlike density of the atomic nucleus, $\delta \rho=m_{N} \delta^{(3)}$, centered at the origin and depending on the nuclear mass $m_{N}$. In spherical coordinates, the static scalar field perturbation then satisfies

$$
\frac{d^{2} \delta \phi}{d r^{2}}+\frac{2}{r} \frac{d \delta \phi}{d r}=-\frac{E^{2}}{M_{\gamma}}+\frac{m_{N}}{M_{m}} \delta^{(3)},
$$

where we have neglected the scalar mass term. This approximation is valid as long as the range of the scalar force $1 / m_{\phi}$ is larger than the size of the atom, implying that $m_{\phi}$ 
must be smaller than $10^{4} \mathrm{eV}$. The solution which vanishes far away from the nucleus is

$$
\delta \phi=-\frac{m_{N}}{4 \pi M_{m} r}-\frac{Z^{2} \alpha}{8 \pi M_{\gamma} r^{2}} .
$$

The sign of the scalar wave function is crucial as it leads to a negative contribution to the energy levels. Notice that, except extremely close to the nucleus, the first contribution dominates over the second one. For example, for a hydrogen atom the first term dominates when $r>10^{-8}\left(M_{m} / M_{\gamma}\right) a_{0}$, with $a_{0}$ the Bohr radius. However, we retain the second term as we will find that the Lamb shift is independent of the scalar field perturbation due to the mass of the nucleus.

At low energy, and in the nonrelativistic limit, the fermion wave function satisfies a Schrödinger equation with the interaction Hamiltonian given by [28]

$$
\begin{aligned}
H= & \frac{p^{2}}{2 m}+W+m-\frac{1}{2 m M_{m}}\left(\delta \phi p^{2}+(\sigma \cdot p) \delta \phi(\sigma \cdot p)\right) \\
& +\frac{m}{M_{m}} \delta \phi,
\end{aligned}
$$

where $m$ is the unperturbed fermion mass and $W$ is a potential describing the interactions of the fermion with all the other fields in the theory. An order of magnitude estimate shows that the dominant perturbation due to the scalar field is

$$
\delta H=\frac{m}{M_{m}} \delta \phi .
$$

We consider the effect of the scalar field on the $l=1$ and $l=2$ energy levels of a hydrogenic atom with nuclear charge $Z e$. The wave functions for these states are (in spherical polar coordinates)

$$
\begin{gathered}
\psi_{1 s}=\frac{1}{\sqrt{\pi}}\left(\frac{Z}{a_{0}}\right)^{3 / 2} e^{-\left(\mathrm{Zr} / a_{0}\right)}, \\
\psi_{2 p}=\frac{1}{\sqrt{\pi}}\left(\frac{Z}{2 a_{0}}\right)^{5 / 2} e^{-\left(Z r / 2 a_{0}\right)} r \cos \theta, \\
\psi_{2 s}=\frac{1}{4 \sqrt{2 \pi}}\left(\frac{Z}{a_{0}}\right)^{3 / 2}\left(2-\frac{Z r}{a_{0}}\right) e^{-\left(Z r / 2 a_{0}\right)},
\end{gathered}
$$

where $a_{0}=\hbar / m_{f} c \alpha$ is the Bohr radius, with $m_{f}$ the mass of the fermion in the orbital, $c$ the speed of light, and $\alpha$ the fine structure constant. The corresponding energy levels are shifted by the effects of the scalar field:

$$
\begin{gathered}
\delta E_{1 s}=-\frac{Z m_{N}}{4 \pi M_{m}^{2} a_{0}} m-\frac{Z^{4} \alpha}{4 \pi a_{0}^{2} M_{m} M_{\gamma}} m, \\
\delta E_{2 s}=-\frac{Z m_{N}}{16 \pi M_{m}^{2} a_{0}} m-\frac{Z^{4} \alpha}{32 \pi a_{0}^{2} M_{m} M_{\gamma}} m,
\end{gathered}
$$

$$
\delta E_{2 p}=-\frac{Z m_{N}}{16 \pi M_{m}^{2} a_{0}} m-\frac{Z^{4} \alpha}{96 \pi a_{0}^{2} M_{m} M_{\gamma}} m .
$$

Notice that the higher levels are less affected by the scalar field but that the gap between the levels has increased. We will study two main effects: the energy gap between the $1 \mathrm{~s}$ and $2 s$ levels and the Lamb shift which involves the difference of energy between the $2 s$ and $2 p$ levels. It is clear from Eqs. (21)-(23) that the Lamb shift is sensitive only to the electric field contribution to the scalar wave function and that the gap between the $1 s$ and $2 s$ levels is essentially due to the nuclear point mass.

\section{A. Precision measurements of hydrogenic atoms}

A strong constraint on $M_{m}$ can be deduced using the precision measurements of hydrogenic energy levels, as a low value of $M_{m}$ would lead to large observable shifts. The $1 s-2 s$ transition for a standard hydrogen atom has a total uncertainty (experimental and theoretical) of order $10^{-9} \mathrm{eV}$ at the $1-\sigma$ level $[32,35,36]$. This transition receives a contribution from the scalar field:

$$
\delta E_{1 s-2 s}=\frac{3 m_{N}}{16 \pi M_{m}^{2} a_{0}} m_{e}+\frac{7 \alpha}{32 \pi a_{0}^{2} M^{2}} m_{e},
$$

where we have defined $M^{2}=M_{m} M_{\gamma}$. The contribution of the term sourced by the atomic electric field satisfies the bound if $\mathrm{GeV} \lesssim M$. However, the nuclear mass term exceeds the $10^{-9} \mathrm{eV}$ bound unless

$$
M_{m} \gtrsim 10 \mathrm{TeV} .
$$

\section{B. The Lamb shift and the proton radius}

A second crucial effect of the coupling of the fermions to the scalar field is the change in the energy difference between levels with differing angular momentum $l$. The most important case is $l=2$, and the contribution to the Lamb shift is

$$
\delta E_{2 s-2 p}=\frac{Z^{4} \alpha}{48 \pi a_{0}^{2} M^{2}} m .
$$

The change in the Lamb shift induced by the scalar field will vary between electronic and muonic atoms. For $Z=1$, we find that for an electron with mass $m_{e}=0.51 \mathrm{MeV}$ and Bohr radius $5.3 \times 10^{-11} \mathrm{~m}$ the scalar contribution to the Lamb shift is

$$
\delta E_{2 s-2 p}\left(e^{-}\right)=3 \times 10^{-10}\left(\frac{\mathrm{GeV}}{M}\right)^{2} \mathrm{eV} .
$$

For a muon of mass $m_{\mu}=106 \mathrm{MeV}$ and Bohr radius $2.5 \times 10^{-13} \mathrm{~m}$ the contribution to the Lamb shift is

$$
\delta E_{2 s-2 p}\left(\mu^{-}\right)=3 \times 10^{-3}\left(\frac{\mathrm{GeV}}{M}\right)^{2} \mathrm{eV} .
$$


As discussed in the introduction the Lamb shift can be used to infer the charge radius of the proton measured in femtometers [37]:

$$
\frac{\Delta E}{\mathrm{meV}}=210-5.23\left(\frac{r_{p}}{\mathrm{fm}}\right)^{2}+0.035\left(\frac{r_{p}}{\mathrm{fm}}\right)^{3} .
$$

Measurements of the Lamb shift may give different values for the charge radius depending on whether the experiments are conducted with electronic or muonic atoms. The Committee on Data for Science and Technology value $r_{p}=0.8768 \pm 0.0069 \mathrm{fm}$ [38] is extracted mainly from spectroscopy of electronic hydrogen atoms and is in agreement with the calculations of bound state quantum electrodynamics $[39,40]$. Ensuring that the electronic Lamb shift lies within the current experimental limits requires $10^{-4} \mathrm{GeV} \lesssim M$. Then the muonic Lamb shift corresponds to a negative variation of the proton radius:

$$
\frac{\delta r_{p}\left(\mu^{-}\right)}{r_{p}}=-0.4\left(\frac{\mathrm{GeV}}{M}\right)^{2},
$$

so that the proton charge radius could vary between measurements with muons and with electrons.

The recent measurement of the proton charge radius for muonic hydrogen [37] gives $r_{p}=0.84184 \mathrm{fm}$ although we note that it may yet be possible to explain this seemingly anomalous measurement with conventional QED [41] and QCD [42]. This corresponds to a negative variation of order $4 \%$ and would require a suppression scale

$$
M \approx 3.2 \mathrm{GeV} .
$$

This is a reasonable scale for an effective theory at low energy which includes protons in its spectrum. Of course, larger values of $M$ lead to a smaller contribution to the proton radius. The bound on $M_{m}$ obtained in (25) is much larger than the value of the averaged scale $M$ deduced from the proton radius deviation, implying $M_{\gamma} \lesssim 10^{-3} \mathrm{GeV}$. In the following section we analyze whether such values are compatible with high energy particle physics experiments.

The Lamb shift for hydrogenic atoms can also be used to constrain $M$. For $Z=2$, the 2- $\sigma$ theoretical and experimental uncertainty is $3 \times 10^{-9} \mathrm{eV}$ [43], while for $Z=15$ it is $6 \times 10^{-4} \mathrm{eV}[44,45]$ at the $1-\sigma$ level and the theoretical uncertainty is $8 \mathrm{eV}$ for $Z=110$ [45]. For $M=$ $3.2 \mathrm{GeV}$, we find that the scalar contributions are respectively $5 \times 10^{-10} \mathrm{eV}, 2 \times 10^{-6} \mathrm{eV}$, and $4 \times 10^{-4}$, which are within these bounds. Hence we find that the constraint on $M$ coming from the proton radius of muonic atoms is compatible with high precision atomic tests for hydrogenic atoms. Larger values of $M$ would lead to even smaller contributions from the scalar field.

We have obtained a strong constraint on $M_{m}$ from the $1 s-2 s$ energy gap of the hydrogen atom, which implies a constraint on $M_{\gamma}$ through measurements of the charge radius of muonic hydrogen. Independent constraints on $M_{\gamma}$ can be deduced from optical cavity experiments and astrophysical observations which probe near vacuum environments. Optical cavity experiments constrain scalar fields with $m_{\phi} \lesssim \mathrm{meV}$ to have $M_{\gamma} \gtrsim 10^{7} \mathrm{GeV}$ $[12,14,18,46]$. Astrophysics constrains $M_{\gamma} \gtrsim 10^{9} \mathrm{GeV}$ for masses of the scalar less than $10^{-12} \mathrm{eV}$ in the interstellar medium [22]. Stronger constraints from helioscope experiments and the alteration of the star burning rate would apply if scalars were produced in the very dense environment inside stars. However, the dependence of the properties of the scalar field on the density of its environment, as in (2), implies that scalars are difficult to produce inside stars. This was first noticed in $[27,47]$ in the chameleon context. In fact as shown in [48] the production of scalars inside a dense plasma can only be realized when the mass of the scalar is tuned to be resonant with the plasma frequency. In other cases, scalars are most likely to be very difficult to produce in stellar plasmas. For this reason, we only consider here constraints on $M_{\gamma}$ coming from near vacuum experiments, and therefore for scalar fields with masses in vacuum $m_{\phi} \gtrsim 10^{-3} \mathrm{eV}$ a coupling $M_{\gamma}<10^{-3} \mathrm{GeV}$ as required to explain the charge radius of muonic hydrogen is permitted by optical experiments.

\section{Anomalous magnetic moment, the $Z$ width, and electroweak precision tests}

So far, we have only considered atomic precision tests. In this subsection, we will confront the bounds deduced from atomic physics with the ones obtained in particle physics. A very stringent particle physics precision test which could be affected by the coupling of scalar fields to fermions is the anomalous magnetic moment $g_{f}-2$ [49]. Typically, the measurement of the anomalous moment of fermions such as the muon involves the decay of pions via weak interactions with resulting $\mathcal{O}(1) \mathrm{GeV}$ scale muons. Therefore the cutoff of the effective theory describing this decay must lie above the weak scale. For such experiments, the contributions of scalars can be calculated [27]:

$$
g_{f}-2=\frac{1}{(4 \pi)^{2}} \frac{m_{f}^{2}}{M_{m}^{2}} \ln \frac{M_{m}^{2}}{m_{f}^{2}} .
$$

A suppression of $M_{m} \approx 600 \mathrm{GeV}$ would lead to $g_{\mu}-2 \approx$ $3 \times 10^{-9}$ and would explain the discrepancy between the standard model prediction and the measured value of the muonic anomalous magnetic moment $[49,50]$. In practice the constraint $M_{m} \geq 10 \mathrm{TeV}$ obtained from the $1 s-2 s$ gap of hydrogenic atoms is a stronger constraint on the strength of the coupling than that from $g_{f}-2$ of the muon.

Gauge invariance at high energy implies that after electroweak symmetry breaking, the coupling scale to photons and the $Z$ boson are the same at the weak scale. Radiative corrections between the weak scale and the QCD scale will not lead to large effects and the two couplings will be essentially the same. Now the coupling of the $Z$ boson to 
a light scalar implies that the width of the $Z$ boson is affected. Known bounds on the $Z$ width can be applied to the branching ratio [33]:

$$
\frac{\Gamma(Z \rightarrow \phi \bar{f})}{\Gamma(Z \rightarrow f \bar{f})} \approx \frac{1}{80 \pi^{3}} \frac{m_{Z}^{2}}{M_{\gamma}^{2}},
$$

where $f \bar{f}$ is a fermion pair and $m_{Z}$ is the mass of the $Z$ boson. The uncertainty on the width is of order $0.0023 \mathrm{GeV}$ compared to a central value of $2.4952 \mathrm{GeV}$. This leads to a bound $M_{\gamma} \geqslant 60 \mathrm{GeV}$ [33]. A stronger bound can also be deduced from the electroweak precision tests [33]. If standard model particles can radiate into scalar states while participating in some measurable process, then the possibility of large corrections to standard model processes arises. The most precise constraints on such corrections come from measurements of electroweak precision observables at LEP, where the effects of scalar fields appear as oblique corrections to the mixing angle $\theta_{W}$, the mass of the $W$ boson, and cross sections and decay rates in the electroweak sector [33]. It was found that large corrections from new scalar fields are screened by a combination of gauge invariance and the allowed structure of the couplings. The remaining logarithmic corrections bound $M_{\gamma} \gtrsim \mathrm{TeV}$. This implies the bound $M \gtrsim 3 \mathrm{TeV}$ meaning that a scalar coupling to both matter and photons cannot explain the observed discrepancy in the proton radius. At most we find that the effect of scalar on the proton radius must be

$$
\left|\frac{\delta r_{p}\left(\mu^{-}\right)}{r_{p}}\right| \leq 10^{-6}
$$

Of course, this is a completely unobservable result.

\section{CONCLUSIONS}

We have shown that scalar fields coupled to matter will shift atomic energy levels. Combinations of atomic precision measurements of electric and muonic hydrogen can be used to probe both the coupling of the scalar field to matter and to photons. The shifts follow from the form of the coupling of scalars to fermions and photons, i.e. a nonrenormalizable operator with a suppression scale which strongly depends on the cut off energy below which the effective Lagrangian description is valid. It also depends on the two types of sources for the scalar field perturbation inside an atom: the nuclear energy density and the nuclear electric field. We find that only the contribution from the electric field has a direct effect on the Lamb shift but that the nuclear energy density has an effect on the $1 s-2 s$ energy gap of hydrogenic atoms.
Particle physics measurements at accelerators have previously been shown to require a large suppression scale for the coupling of the scalar field to the gauge sector of the standard model. We have shown that the constraint deduced from measurements of the $1 s-2 s$ energy gap of the hydrogen atom is stronger and constrains $M_{m} \gtrsim 10 \mathrm{TeV}$. We have obtained that the scalar field perturbation of the Lamb shift is sensitive only to the product of the coupling scales $M_{m} M_{\gamma}$ and that the scalar field perturbation is much larger for muonic that electric hydrogen atoms. However, previously derived constraints on $M_{\gamma}$ coming from the electroweak precision tests, $M_{\gamma} \gtrsim 1 \mathrm{TeV}$, imply that the anomalous measurement of the muonic Lamb shift cannot be explained through the presence of a scalar field. This would have required a low value of the coupling scale of the scalar to photons $M_{\gamma} \sim \mathrm{MeV}$.

In summary, we have shown that the best universal atomic and particle physics bounds on the coupling scales of matter and photons to a scalar field with mass $10^{-3} \mathrm{eV} \lesssim m_{\phi} \lesssim 10^{3} \mathrm{eV}$ are a new bound on the coupling to matter $M_{m} \gtrsim 10 \mathrm{TeV}$ from energy level shifts in the hydrogen atom and the previously determined bound $M_{\gamma} \gtrsim 1 \mathrm{TeV}$ from electroweak precision tests. For light scalar fields with fixed masses, $\lesssim 10^{-3} \mathrm{eV}$, and couplings the measurements of fifth force searches already exclude all coupling to matter up to and beyond the Planck scale, and optical experiments in the laboratory and astrophysics lead to tighter bounds on the coupling to photons $M_{\gamma} \gtrsim 10^{10} \mathrm{GeV}$. However, these constraints can be easily avoided in models such as the chameleon and Galileon theories by nonlinear mechanisms. In contrast the constraints discussed in this article are universal and should be considered as a minimal set of requirements on any coupled scalar with mass $10^{-3} \mathrm{eV} \lesssim m_{\phi} \lesssim 10^{3} \mathrm{eV}$. Although we have found that the Lamb shift in hydrogenic and muonic atoms differs due to the difference of mass between the electron and the muon, the large values of $M_{m}$ and $M_{\gamma}$ imply that the shift in the proton radius due to a scalar field is negligible. Therefore it seems that the best possibility of detecting a coupled scalar field in laboratory experiments is not with atomic measurements but with optical cavity experiments.

\section{ACKNOWLEDGMENTS}

We thank Andreas Ringwald for very helpful discussions in the preparation of this work. C. B. was supported by the German Science Foundation (DFG) under the Collaborative Research Centre (SFB) 676 and by the SNF. 
[1] P. Svrcek and E. Witten, J. High Energy Phys. 06 (2006) 051.

[2] A. Arvanitaki, S. Dimopoulos, S. Dubovsky, N. Kaloper, and J. March-Russell, Phys. Rev. D 81, 123530 (2010).

[3] J. Jaeckel and A. Ringwald, Annu. Rev. Nucl. Part. Sci. 60, 405 (2010).

[4] C. M. Will, Living Rev. Relativity 9, 3 (2006), http:// www.livingreviews.org/lrr-2006-3.

[5] J. Khoury and A. Weltman, Phys. Rev. D 69, 044026 (2004).

[6] J. Khoury and A. Weltman, Phys. Rev. Lett. 93, 171104 (2004).

[7] A. Nicolis, R. Rattazzi, and E. Trincherini, Phys. Rev. D 79, 064036 (2009).

[8] S. Schlamminger, K. Y. Choi, T.A. Wagner, J.H. Gundlach, and E. G. Adelberger, Phys. Rev. Lett. 100, 041101 (2008).

[9] P. Brax, C. Burrage, A.-C. Davis, D. Seery, and A. Weltman, arXiv:1010.4536.

[10] P. Brax, C. Burrage, A.-C. Davis, D. Seery, and A. Weltman, Phys. Rev. D 81, 103524 (2010).

[11] G. Raffelt and L. Stodolsky, Phys. Rev. D 37, 1237 (1988).

[12] R. Cameron, G. Cantatore, A. C. Melissinos, G. Ruoso, Y. Semertzidis, H. J. Halama, D. M. Lazarus, A. G. Prodell, F. Nezrick, C. Rizzo, and E. Zavattini, Phys. Rev. D 47, 3707 (1993).

[13] C. Robilliard et al., Phys. Rev. Lett. 99, 190403 (2007).

[14] A. S. Chou et al. [GammeV (T-969) Collaboration], Phys. Rev. Lett. 100, 080402 (2008).

[15] P. Pugnat, L. Duvillaret, R. Jost, G. Vitrant, D. Romanini, A. Siemko, R. Ballou, B. Barbara, M. Finger, M. Finger, J. Hošek, M. Král, K. A. Meissner, M. Šulc, and J. Zicha (OSQAR Collaboration), Phys. Rev. D 78, 092003 (2008).

[16] M. Fouche et al., Phys. Rev. D 78, 032013 (2008).

[17] A. Afanasev et al., Phys. Rev. Lett. 101, 120401 (2008).

[18] K. Ehret, M. Frede, S. Ghazaryan, M. Hildebrandt, E.-A. Knabbe, D. Kracht, A. Lindner, J. List, T. Meier, N. Meyer, D. Notz, J. Redondo, A. Ringwald, G. Wiedemann, and B. Willke, Phys. Lett. B 689, 149 (2010).

[19] G. G. Raffelt, Lect. Notes Phys. 741, 51 (2008).

[20] A. De Angelis, O. Mansutti, and M. Roncadelli, Phys. Rev. D 76, 121301 (2007).

[21] E. Arik et al. (CAST Collaboration), J. Cosmol. Astropart. Phys. 02 (2009) 008.

[22] C. Burrage, A.-C. Davis, and D. J. Shaw, Phys. Rev. D 79, 044028 (2009).

[23] C. Burrage, A.-C. Davis, and D. J. Shaw, Phys. Rev. Lett. 102, 201101 (2009).

[24] M. Fairbairn, T. Rashba, and S. V. Troitsky, arXiv:0901.4085.

[25] A. Mirizzi and D. Montanino, J. Cosmol. Astropart. Phys. 12 (2009) 004
[26] N. Bassan, A. Mirizzi, and M. Roncadelli, J. Cosmol. Astropart. Phys. 05 (2010) 010.

[27] P. Brax, C. van de Bruck, and A.-C. Davis, Phys. Rev. Lett. 99, 121103 (2007).

[28] P. Brax and C. Burrage, Phys. Rev. D 82, 095014 (2010).

[29] W.E. Lamb and R. C. Retherford, Phys. Rev. 72, 241 (1947).

[30] Effects of the finite size of the proton are relevant for $s$ states, because the wave function does not vanish at the location of the proton, whereas $p$ states remain unaffected, and so the difference in energy between the $2 s$ and $2 p$ energy levels is sensitive to the dimensions of the proton. Muonic hydrogen is more sensitive to this effect than electronic hydrogen because the heavier mass of the muon corresponds to a much smaller Bohr radius.

[31] M. Pospelov, Phys. Rev. D 80, 095002 (2009).

[32] J. Jaeckel and S. Roy, Phys. Rev. D 82, 125020 (2010).

[33] P. Brax, C. Burrage, A.-C. Davis, D. Seery, and A. Weltman, J. High Energy Phys. 09 (2009) 128.

[34] L. Hui and A. Nicolis, Phys. Rev. Lett. 105, 231101 (2010).

[35] C. Schwob et al., Phys. Rev. Lett. 82, 4960 (1999).

[36] G. G. Simon, C. Schmitt, F. Borkowski, and V. H. Walther, Nucl. Phys. A333, 381 (1980).

[37] R. Pohl et al., Nature (London) 466, 213 (2010).

[38] P. J. Mohr, B. N. Taylor, and D. B. Newell, Rev. Mod. Phys. 80, 633 (2008).

[39] M. I. Eides, H. Grotch, and V. A. Shelyuto, Phys. Rep. 342, 63 (2001).

[40] S. G. Karshenboim, Phys. Rep. 422, 1 (2005).

[41] A. De Rujula, Phys. Lett. B 693, 555 (2010).

[42] T. Friedmann, arXiv:0910.2231.

[43] A. van Wijngaarden, J. Kwela, and G. W. F. Drake, Phys. Rev. A 43, 3325 (1991).

[44] D. Müller, J. Gassen, L. Kremer, H.-J. Pross, F. Scheuer, H.-D. Sträter, P. von Brentano, A. Pape, and J. C. Sens, Europhys. Lett. 5, 503 (1988).

[45] W. R. Johnson, I. Bednyakov, and G. Soff, Phys. Rev. Lett. 87, 233001 (2001).

[46] These results have been recently extended to a larger mass range up to $0.1 \mathrm{eV}$ by the GammeV-CHASE group. For masses larger than $10^{-3} \mathrm{eV}$, the bound on $M$ becomes looser and looser up to a scale of order $0.1 \mathrm{eV}$.

[47] J. Jaeckel, E. Masso, J. Redondo, A. Ringwald, and F. Takahashi, Phys. Rev. D 75, 013004 (2007).

[48] P. Brax and K. Zioutas, Phys. Rev. D 82, 043007 (2010).

[49] F. Jegerlehner, Acta Phys. Pol. B 38, 3021 (2007).

[50] K. Hagiwara, A. D. Martin, D. Nomura, and T. Teubner, Phys. Lett. B 649, 173 (2007). 